\title{
Front Matter: Volume 11079
}

, "Front Matter: Volume 11079," Proc. SPIE 11079, Medical Laser Applications and Laser-Tissue Interactions IX, 110791Y (2 October 2019); doi:

10.1117/12.2544417

SPIE. Event: European Conferences on Biomedical Optics, 2019, Munich, Germany 


\section{PROGRESS IN BIOMEDICAL OPTICS AND IMAGING}

\section{Medical Laser Applications and Laser-Tissue Interactions IX}

Lothar D. Lilge

Carsten M. Philipp

Editors

\section{3-24 June 2019}

Munich, Germany

Sponsored by

The Optical Society (United States)

SPIE

Published by

SPIE 
The papers in this volume were part of the technical conference cited on the cover and title page. Papers were selected and subject to review by the editors and conference program committee. Some conference presentations may not be available for publication. Additional papers and presentation recordings may be available online in the SPIE Digital Library at SPIEDigitalLibrary.org.

The papers reflect the work and thoughts of the authors and are published herein as submitted. The publisher is not responsible for the validity of the information or for any outcomes resulting from reliance thereon.

Please use the following format to cite material from these proceedings:

Author(s), "Title of Paper," in Medical Laser Applications and Laser-Tissue Interactions IX, edited by Lothar D. Lilge, Carsten M. Philipp, Proc. of SPIE-OSA Vol. 11079 (SPIE, Bellingham, WA, 2019) Sevendigit Article CID Number.

ISSN: 1605-7422

ISSN: 2410-9045 (electronic)

ISBN: 9781510628519

ISBN: 9781510628526 (electronic)

Copublished by

SPIE

P.O. Box 10, Bellingham, Washington 98227-0010 USA

Telephone +1 3606763290 (Pacific Time) · Fax +1 3606471445

SPIE.org

and

The Optical Society

2010 Massachusetts Ave., N.W., Washington, D.C., 20036 USA

Telephone 1 202/223-8130 (Eastern Time) · Fax 1 202/223-1096

http://www.osa.org

Copyright (@ 2019, Society of Photo-Optical Instrumentation Engineers and The Optical Society

Copying of material in this book for internal or personal use, or for the internal or personal use of specific clients, beyond the fair use provisions granted by the U.S. Copyright Law is authorized by SPIE subject to payment of copying fees. The Transactional Reporting Service base fee for this volume is $\$ 21.00$ per article (or portion thereof), which should be paid directly to the Copyright Clearance Center (CCC), 222 Rosewood Drive, Danvers, MA 01923. Payment may also be made electronically through CCC Online at copyright.com. Other copying for republication, resale, advertising or promotion, or any form of systematic or multiple reproduction of any material in this book is prohibited except with permission in writing from the publisher. The CCC fee code is 1605 $7422 / 19 / \$ 21.00$.

Printed in the United States of America by Curran Associates, Inc., under license from SPIE.

Publication of record for individual papers is online in the SPIE Digital Library.

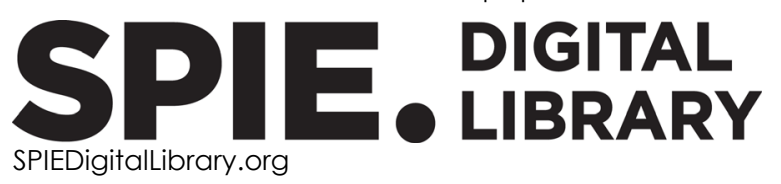

Paper Numbering: Proceedings of SPIE follow an e-First publication model. A unique citation identifier (CID) number is assigned to each article at the time of publication. Utilization of CIDs allows articles to be fully citable as soon as they are published online, and connects the same identifier to all online and print versions of the publication. SPIE uses a seven-digit CID article numbering system structured as follows:

- The first five digits correspond to the SPIE volume number.

- The last two digits indicate publication order within the volume using a Base 36 numbering system employing both numerals and letters. These two-number sets start with 00, 01, 02, 03, 04, 05, 06, 07, 08, 09, OA , OB ... 0Z, followed by 10-1Z, 20-2Z, etc. The CID Number appears on each page of the manuscript. 


\title{
Contents
}

\author{
ix Authors \\ xiii Conference Committee
}

POSTER SESSION

1107901 Distribution between spatial and spectral kinds of information in the microscopy systems which perform multispectral processing of biological objects images [11079-68]

\section{PULSED LASER AND PHOTOTHERMAL APPLICATIONS I}

1107902 Dosimetry for microsecond selective laser trabeculoplasty (Invited Paper) [11079-1]

1107903 Optical coherence tomography controlled selective retina therapy with a novel microsecond laser [11079-2]

1107906 Biomechanical FEM model of the cornea in femtosecond laser assisted keratoplasty [1 1079-5]

\section{SHORT PULSE AND PHOTOTHERMAL APPLICATIONS II}

$1107907 \quad$ Ex vivo efficacy demonstration of a laser fenestration system for endovascular abdominal aortic aneurysm repair (EVAR) [1 1079-6]

$1107908 \quad$ Noncontact tagging and identification of preimplantation mammalian embryos by means of ultrafast laser microsurgery [1 1079-7]

1107909 Investigations on thermography in laser medicine [1 1079-8]

11079 OA A mid-infrared laser features and fat reduction efficacy [1 1079-9]

11079 OB Toward feedback temperature control for retinal laser treatment [1 1079-10]

11079 OC Low-temperature (cavitations) dissection of biological tissues by quasi-continuous laser irradiation [1 1079-11] 
11079 OD Two-channel portable fluorescence meter for risk stratification of cardiovascular diseases [11079-12]

11079 OE Rapid spectrophotometric quantification of urinary porphyrins and porphobilinogen as screening tool for attacks of acute porphyria [1 1079-13]

11079 OF An in vivo two photon fluorescence endomicroscopic probe based on a 2-axis electrothermal MEMS mirror [1 1079-14]

11079 OG Clinical translation of Raman-based multimodal spectral histopathology for margin assessment during surgery of basal cell carcinoma [11079-15]

11079 ol Clinical spectroscopy for biotissues and bioliquids [1 1079-17]

$110790 \mathrm{~J} \quad$ Bringing third and second harmonic generation microscopy into the clinic for the assessment of fresh lung tissue [1 1079-18]

\section{PDT DELIVERY AND MONITORING I}

11079 OK Optical coherence angiography monitoring of tumor early response to PDT in experimental and clinical studies (Invited Paper) [1 1079-19]

$11079 \mathrm{OL} \quad$ Evaluation of singlet oxygen production of a novel chlorin photosensitizer [11079-20]

11079 OM Transbronchial light illumination for peripheral lung cancer: a numerical feasibility study [11079-21]

\section{PDT DELIVERY AND MONITORING II}

11079 ON ALA/PpIX photodiagnosis of stress-induced gastrointestinal primary tumors and metastases in experimental animals [11079-22]

$1107900 \quad$ Monitoring of photodynamic therapy with target nanoconstructs by fluorescence and optoacoustic imaging: numerical simulations and phantom study [1 1079-23]

11079 OP Individualization of interstitial photodynamic therapy for malignant gliomas [1 1079-25]

$110790 Q \quad$ Increase and homogenization of the endogenous production of protoporphyrin IX by photobiomodulation [1 1079-27]

11079 OR Red and blue light photodynamic therapy regimes: optical monitoring and histology studies [11079-26] 
11079 OS Blue light induced modulation in the early phase of wound healing [1 1079-28]

11079 ОT Action of He-Ne laser on wounded human skin fibroblast cells [1 1079-29]

11079 OU Can photobiomodulation therapy be an alternative to pharmacological therapies in decreasing the progression of skeletal muscle impairments of mdx mice? [11079-30]

11079 OV Human keloid cultured fibroblasts irradiated with blue LED light: evidence from an in vitro study [11079-31]

11079 OW Heating and optoacoustic temperature determination of cell cultures [11079-32]

$110790 \mathrm{X}$ Endocardial irrigated catheter for volumetric optoacoustic mapping of radio-frequency ablation [1 1079-33]

\section{SPECTROSCOPY AND OTHER BIOPHOTONICS TECHNOLOGIES}

$110790 Z$ Femtosecond laser printing of living human cells [1 1079-35]

$1107910 \quad$ FullMonte: fast Monte-Carlo light simulator [1 1079-36]

$1107911 \quad$ Laser irradiation induces mitochondrial dysfunction in hepatic cells [11079-37]

1107912 Radiation profile measurement methods for optical light diffusers: comparison of imaging and non-imaging methods [1 1079-38]

1107913 Standardization phantom for intra-operative fluorescence molecular imaging [11079-39]

\section{POSTER SESSION}

1107914 Characterising UV transmission property of red hair using microspectrophotometer [11079-40]

1107915 Debonding of ceramic brackets using line laser system [1 1079-41]

1107916 Near-real time monitoring of bacterial viability using the optrode: a portable fluorimeter [11079-42]

1107918 Assessment of age-related skin changes using hyperspectral polarization imaging [11079-44]

1107919 Quantitative analysis of portwine stainbirthmark color in PDT treatment [1 1079-45] 
11079 1A Spectroscopic discrimination of green tea's impact on teeth at different temperatures [1 1079-46]

11079 1B Laser excitation of acoustic pulses in absorbing and scattering tissues: numerical solution of three-dimensional problems [11079-47]

11079 1C Optical fine-needle biopsy approach for intraoperative multimodal diagnostics in minimally invasive abdominal surgery [1 1079-48]

11079 1D Cross-polarization OCT needle probe for combined blood vessels detection and tissue differentiation during stereotactic biopsy of brain tumors [1 1079-49]

11079 1E Synergistic effects of combination of three wavelengths and different light sources in cytochrome c oxidase activity in intact skeletal muscle of rats [1 1079-50]

11079 IF Near-infrared light therapy for recovery of cerebral hypoperfusion induced by bilateral common carotid artery stenosis in mice [11079-51]

$110791 \mathrm{H} \quad$ Ex vivo investigation of different $\mu$ s laser pulse durations for selective retina therapy [1 1079-53]

$1107911 \quad$ Dual-wavelength fluorescence localization of chlorin-based photosensitizer [1 1079-54]

$110791 \mathrm{~J} \quad$ Selective damages of tumor vessels by $1060 \mathrm{~nm}$ pulsed irradiation [1 1079-55]

$110791 \mathrm{~K} \quad$ Comparative analysis of single- and dual-wavelength low dose photodynamic therapy regimens [1 1079-57]

$110791 \mathrm{~L} \quad$ Solid state versus fiber picosecond infrared lasers applied to two-photon vision tests [1 1079-58]

11079 1M Hydrogen peroxide level in tumor cells during cisplatin-induced apoptosis [1 1079-59]

$110791 \mathrm{~N} \quad$ Simulations for modeling the photothermal response of nerve tissue [1 1079-60]

$1107910 \quad$ Wearable sensor system for multipoint measurements of blood perfusion: pilot studies in patients with diabetes mellitus [1 1079-62]

$110791 Q \quad$ Analysis of changes in blood flow oscillations under different probe pressure using laser Doppler spectrum decomposition [1 1079-64]

11079 IR Nd:YAG laser on dental enamel in the reduction of artificial caries demineralization [1 1079-61]

11079 is Optical methods for differential diagnostics between secondary bladder cancer and late adverse events after pelvic radiation therapy [11079-56]

11079 1T Ceruloplasmin: a potential carrier of photosensitizers for photodynamic therapy of tumors [1 1079-24]

11079 IW Multidimensional images processing in electrocardiography using Bragg diffraction technologies [11079-67] 
$110791 \mathrm{X}$ The study of focused ultrasound effect to increase the penetration of light for photodynamic therapy of deeper tissue [11079-69]

Proc. of SPIE-OSA Vol. 11079 110791Y-7

Downloaded From: https://www.spiedigitallibrary.org/conference-proceedings-of-spie on 26 Apr 2023 Terms of Use: https://www.spiedigitallibrary.org/terms-of-use 
Proc. of SPIE-OSA Vol. 11079 110791Y-8

Downloaded From: https://www.spiedigitallibrary.org/conference-proceedings-of-spie on 26 Apr 2023 Terms of Use: https://www.spiedigitallibrary.org/terms-of-use 


\section{Authors}

Numbers in the index correspond to the last two digits of the seven-digit citation identifier (CID) article numbering system used in Proceedings of SPIE. The first five digits reflect the volume number. Base 36 numbering is employed for the last two digits and indicates the order of articles within the volume. Numbers start with 00, 01, 02, 03, 04, 05, 06, 07, 08, 09, 0A, OB...0Z, followed by 10-12, 20-2Z, etc.

Abbas, H. S., OB

Abi Haidar, D., OF

Afsharnejad, Yasmin, 10

Agranovich, llana, ON

Ahmed, Irfan, 1A

Ahn, Jin-Chul, 15

Alekseyev, Alexander, 1C

Alfieri, Domenico, OS

Al-Jumaily, Ahmed M., 14

Anastasopoulou, Maria, 13

Angelov, Ivan, $0 \mathrm{~N}$

Annema, Jouke T., 0J

Arnold, Patrik, 03

Artyushenko, Viacheslav, 01

Aumiller, Maximilian, OP

Bacci, Stefano, OS

Bahce, Idris, OJ

Balalaeva, Irina $\vee ., 1 \mathrm{M}$

Banchelli, Martina, OV

Belikova, Valeria, 01

Berchiolli, Raffaella Nice, 07

Betz, Vaughn, 0M, 10

Bibikova, Olga, 0 I

Bliedtner, Katharina, 02

Bogdanov, Alexey A., $1 \mathrm{~J}$

Boitor, Radu, OG

Borisova, Ekaterina, $\mathrm{ON}$

Bourko, Vladimir D., OC

Bouvet, F., OF

Brinkmann, Ralf, 02, 03, OB, OW, $1 \mathrm{H}$

Burri, Christian, 03, 1H

Byers, Patrick, $\mathrm{OZ}$

Bykov, Alexander, 18

Canovetti, Annalisa, 06

Castro, Pedro A. A., IR

Chalmers, Andrew N., 14

Chang, Won Seok, $1 \mathrm{X}$

Cheng, Shuk Han, IA

Cherchi, Federica, OV

Choi, Woo Sung, IF

Clausen-Schaumann, Hauke, $\mathrm{OZ}$

Condino, Sara, 07

Coppi, Elisabetta, OV

Cypel, Marcelo, OM

da Silva Tomazoni, Shaiane, OU, IE

Danicke, V., OB

Daniels, Johannes M. A., OJ

Danielyan, Georgy, 0 I

De Siena, Gaetano, OS
De Simone, Paolo, 07

Deán-Ben, Xosé Luis, OX

Dejneka, Alexandr, 11

Detrez, Nicolas, OW

Dickhoff, Chris, 0J

Ding, Rui, OL

Do, Woo Jong, 15

Docheva, Denitsa, $0 Z$

Domke, M., 12

Dong, Kui, 19

Dremin, Viktor V., 18, 1C, 1Q

Dudenkova, $V$. $\vee .$, is

Dunaev, Andrey V., 1C, 10, 1Q

Dzhagarov, Boris M., IT

Ebneter, Andreas, 03

Egorova, Daria, 11

Eisel, Maximilian, 09, OP

Esnault, C., OF

Feldchtein, Felix I., OK

Feliksberger, Elena, $0 \mathrm{l}$

Ferrara, Paolo, 06

Ferrari, Mauro, 07

Ferrari, Vincenzo, 07

Filatov, Maxim A., 08

Fraccalvieri, Marco, OV

Frank, Christine, $\mathrm{OZ}$

Freymüller, Christian, 09, OP

Fu, Xiang, 14

Gasperini, Stefano, OS, OV

Gelikonov, Grigory V., ID

Genova, Tsanislava, ON

Gerelli, Emmanuel, $\mathrm{OQ}$

Gladkova, Natalia D., OK, ID

Goncalves, Sergio E. P., IR

Gorpas, Dimitris, 13

Grabovskis, Andris, 18

Grimm, Lorenz, 03

Grishanov, Vladimir N., OD

Groot, Marie Louise, OJ

Gubarkova, Ekaterina V., OK

Gurevich, B. S., 01, 1W

Gyulkhandanyan, Anna G., $1 T$

Gyulkhandanyan, Aram G., $1 T$

Gyulkhandanyana, Grigor V., IT

Han, Seung Hee, $1 X$

Han, Zhen, $\mathrm{OL}$

Hartmann, Bastian, $\mathrm{OZ}$

Hasan, T., 00

Heckl, C., OE 
Hennig, G., OE

Herzog, C., OB

Hocotz, Thaddäus, 0

Homann, C., OE

Huang, Xiyong, 14

Huang, Zheng, OL, 19

Huber, Heinz P., OZ

Hung, Tak Fu, $1 \mathrm{~A}$

Hutfilz, Alessa, 03, 1H

llina, Inna $V_{.,} 08$

Jang, Ha-Young, $1 \mathrm{~F}$

Jiang, Jie, OL, 19

Joniová, Jaroslava, $0 Q$

Jung, Gu-In, 15

Kandurova, Ksenia, 1C

Kanevskiy, Matvey, ON

Karev, Vadim E., $1 \mathrm{~J}$

Khilov, Aleksandr V., 0O, 0R, 11, 1K

Khorovodov, Alexander, ON

Khramova, Yulia V., 08

Kienle, Alwin, OP

Kim, Byeong Kwon, 15

Kim, Dae Yu, $1 F$

Kim, Jae Hun, IF

Kim, Jae Hyuk, $1 \mathrm{X}$

Kirillin, Mikhail YU., 0O, OR, 1I, IK

Kiseleva, Elena B., 1D, 1S

Klemm, Uwe, 13

Klimenko, Vladimir $V_{\text {., }} 1 \mathrm{~J}$

Knyazev, Nikolay A., $1 \mathrm{~J}$

Koch, Maximilian, 13

Koloydenko, Alexey, OG

Komar, Katarzyna, $1 \mathrm{~L}$

Kong, Kenny, OG

Konnova, Svetlana, ON

Kopaev, Dmitry E., OD

Kornaszewski, Łukasz, 1L

Kornilin, Dmitriy $\vee ., O D$

Korzhimanova, Yulia V., 1D

Kozlov, Igor O., 1C, 1O, 1Q

Kren, C., OB

Kubinová, Šárka, 11

Kulikov, Andrei, 11

Kurakina, Daria A., 00, 0R, 11, 1K

Kuznetsov, Sergei S., OK

Kwon, Sung-Min, 15

Lang, A., OE

Lau, Condon, $1 \mathrm{~A}$

Lazareva, Ekaterina N., $1 \mathrm{~T}$

Leal-Junior, Ernesto Cesar Pinto, OU, IE

Lebedev, Petr A., OD

Lee, Dong-Jin, $1 \mathrm{~F}$

Lee, Eun-Joo, $1 \mathrm{~F}$

Lee, Ji-Young, OA

Lee, Jong Hoon, 15

Li, Vincent Wai, 1A

Li, Weijun, 19

Liemert, André, OP

Lilge, Lothar, OM, 10

Loktionova, Yulia I., 10
Lunov, Oleg, 11

Lunova, Mariia, 11

Lynnyk, Anna, 11

Magni, Giada, 07, OS, OV

Mahato, Krishna Kishore, OT

Malandrini, Alex, 06

Mallidi, S., 00

Mamoshin, Andrian, $1 \mathrm{C}$

Mangia, Antongiulio, OV

Manno, Francis A. M., IA

Manno, Sinai H. C., 1A

Mantareva, Vanya, ON

Marchi, Gabriele, $\mathrm{OZ}$

Marcinkevics, Zbignevs, 18

Marzejon, Marcin, $1 \mathrm{~L}$

Maslennikova, A. V., is

Matteini, Paolo, OV

Matveev, Lev A., OK

McFadden, Christopher, OM

McGoverin, Cushla, 16

Meglinski, Igor V., 18, 1Q

Mehidine, $\mathrm{H}_{\text {., }} \mathrm{OF}$

Meier, Christoph, 03, $1 \mathrm{H}$

Melenteva, Anastasya, 0 l

Meller, Alina, OR

Menabuoni, Luca, 06

Mezentsev, Mikhail A., 1Q

Micheletti, Filippo, 07

Min, L., OF

Minet, Olaf, Ol

Miura, Yoko, OW

Moiseev, Alexander A., OK

Molenhuis, Daniel, 10

Moon, Ki-Wook, $1 \mathrm{~F}$

Navolokin, Nikita, ON

Nerush, Anastasiya S., $1 \mathrm{M}$

Nieberler, Markus, 13

Notingher, loan, OG

Ntziachristos, Vasilis, 13

Oh, Suk-Won, OA

Orlinskaya, Natalia YU., OR, $1 \mathrm{~K}$

Orlova, Anna G., 00, OR, 1M

Ou, Fang, 16

Özsoy, Çağla, OX

Park, Sungjo, 15

Parkhots, Marina V., $1 T$

Paul, Sharad P., 14

Pavone, Francesco S., OS, OV

Pedata, Felicita, OV

Perekatova, V. V., 00

Piazza, Roberta, 07

Pimenova, Darya Yu., OD

Pini, Roberto, 06, OS, OV

Pinot, L., OF

Plehanov, V. I., 00

Popov, Alexey, 18

Potapova, Elena V., 1C, 1Q

Považay, Boris, 03

Prabhu, Vijendra, 0T

Protheroe, Michael D., 14 
Pugliese, Anna Maria, OV

Radonic, Teodora, OJ

Rafailov, Edik U., 10

Rafailov, llya E., 10

Ramadan, Khaled, OM

Rao, Bola Sadashiva Satish, OT

Razansky, Daniel, OX

Robertson, Julia, 16

Romanov, Oleg G., 0C, 1B

Romashov, Vladimir N., ID

Rossi Degl'Innocenti, Duccio, OS, OV

Rossi, Francesca, 06, 07, 05, OV

Rossi, Michele, 06

Rühm, Adrian, 09, OP

Rusanov, Anatoliy A., $1 \mathrm{~J}$

Ryu, Han-Young, OA

Sakharova, Tatiana, 0 I

Schulte-Spechtel, Levin, $0 Z$

Schwiegelshohn, Fynn, OM, 10

Seifert, Eric, 02, 0W

Seinstra, Daniëlle, 0J

Semyachkina-Glushkovskaya, Oxana, ON

Seo, Young-Seok, OA

Sergeeva, Ekaterina, 00, OR

Seryogina, Evgeniya, 1C

Shakhova, Maria A., OR, $1 \mathrm{~K}$

Shilyagin, Pavel A., 1D

Shupletsov, Valery V., 1C, 1Q

Shchukina, Kseniya M., $1 M$

Sidorov, Victor V., 10

Siegel, Julian, $0 Z$

Silva, Mateus R., $1 R$

Silva, Tânia M., $1 R$

Sirotkina, Marina A., OK, ID

Sitnikov, Dmitry S., 08

Smolková, Barbora, 11

Sokolovski, Sergei G., 10

Sroka, Ronald, 09, OE, OP, 12

Stauch, T., OE

Stepp, Herbert, OE, OP

Streltsova, O.S., 1S

Ströbl, Stephan, 09, 12

Subochev, P. V., 00

Sudhop, Stefanie, $\mathrm{OZ}$

Sung, Chang Kyu, $1 \mathrm{~F}$

Swift, Simon, 16

Tararova, E. A., 1S

Targetti, Lorenzo, OS, OV

Tatini, Francesca, OS

Theisen-Kunde, Dirk, 03, ow

Tian, Li, IA

Tozburun, Serhat, $1 \mathrm{~N}$

Trebst, T., 12

Tripodi, Cristina, OS

Tuchin, Valery V., IT

Turchin, llya, 00, 0R, 11

Turker, Merve, $1 \mathrm{~N}$

Usenov, Iskander, 0

Uzhytchak, Mariia, 11

van Boven, Wim-Jan P., OJ van Huizen, Laura M. G., 0J

van Mourik, Frank, OJ

Vanholsbeeck, Frédérique, 16

Varma, Sandeep, OG

Verlov, Nikolay A., $1 \mathrm{~J}$

Vitkin, Alex, OK

Vogeser, M., OE

Wagnières, Georges, $0 Q$

Wang, Yuzhi, 19

Williams, Hywel C., OG

Wojtkowski, Maciej, $1 \mathrm{~L}$

Xie, H., OF

Yashin, Konstantin S., 1D

Yoo, A-Ram, 1F

Young-Schultz, Tanner, 10

Yuan, Kaihua, 19

Yunusova, K. E., 1S

Zabarylo, Urszula J., Ol

Zagaynova, Elena V., OK, 1D

Zaichenko, K. V., 01, IW

Zaitsev, Vladimir Y., OK

Zakharov, Valeriy P., OD

Zakoyana, Anna A., IT

Zezell, Denise M., IR

Zhang, Jun, $\mathrm{OZ}$

Zharkikh, Elena V., 10

Zheltov, George I., OC, 1B

Zheng, Zhangcheng, $O M$

Zherebtsov, Evgeny A., 18, 1C, 10, 1Q

Zherebtsova, Angelina I., 10, 1Q

Zou, Jian, OL 
Proc. of SPIE-OSA Vol. 11079 110791Y-12

Downloaded From: https://www.spiedigitallibrary.org/conference-proceedings-of-spie on 26 Apr 2023 Terms of Use: https://www.spiedigitallibrary.org/terms-of-use 


\title{
Conference Committee
}

\author{
Symposium Chairs
}

Brett E. Bouma, Wellman Center for Photomedicine (United States)

Paola Taroni, Politecnico di Milano (Italy)

Ronald Sroka, Laser-Forschungslabor (Germany)

I. Alex Vitkin, University of Toronto (Canada)

Conference Chairs

Lothar D. Lilge, Princess Margaret Hospital (Canada)

Carsten M. Philipp, Evangelische Elisabeth Klinik (Germany)

Conference Programme Committee

Christian Stephan Betz, Klinikum der Universität München (Germany)

Ralf Brinkmann, Universität zu Lübeck (Germany)

Santiago Camacho Lopez, Centro de Investigación Científica y de

Educación Superior de Ensenada B.C. (Mexico)

Matthias Domke, FH Vorarlberg (Austria)

Martin Frenz, University Bern (Switzerland)

Zheng Huang, University of Colorado Denver (United States)

Hyun Wook Kang, Pukyong National University (Korea, Republic of)

Mikhail Y. Kirillin, Institute of Applied Physics (Russian Federation)

Igor Meglinski, University of Oulu (Finland)

Serge R. Mordon, INSERM (France)

Angelika C. Rueck, Universität Ulm (Germany)

Adrian Rühm, Laser-Forschungslabor (Germany)

David D. Sampson, The University of Western Australia (Australia)

Herbert Stepp, Klinikum der Universität München (Germany)

Frank Strittmatter, Klinikum der Universität München (Germany)

Valery V. Tuchin, Saratov State University (Russian Federation)

Georges Wagnières, Ecole Polytechnique Fédérale de Lausanne

(Switzerland)

\section{Session Chairs}

1 Pulsed Laser and Photothermal Applications I

Ralf Brinkmann, Medizinisches Laserzentrum Lübeck $\mathrm{GmbH}$

(Germany)

2 Short Pulse and Photothermal Applications II

Adrian Ruehm, LIFE-Zentrum am Klinikum der Universität München (Germany) 
HT ECBO Hot Topics

I. Alex Vitkin, University Health Network (Canada)

Ronald Sroka, Laser-Forschungslabor (Germany)

3 Optical Diagnostic Techniques

Marie Louise Groot, Vrije University Amsterdam (Netherlands)

$4 \quad$ PDT Delivery and Monitoring I

Zheng Huang, University of Colorado Denver (United States)

5 PDT Delivery and Monitoring II

Mikhail Yu. Kirillin, Institute of Applied Physics of the RAS

(Russian Federation)

Lothar D. Lilge, Princess Margaret Cancer Center (Canada)

6 Photobiomodulation and Photoacoustics

Lothar D. Lilge, Princess Margaret Cancer Center (Canada)

7 Spectroscopy and other Biophotonics Technologies

Fynn Schwiegelshohn, University of Toronto (Canada) 